\title{
TRIANGULATED INFINITE-DIMENSIONAL MANIFOLDS
}

\author{
BY DAVID W. HENDERSON ${ }^{1}$ AND JAMES E. WEST ${ }^{2}$
}

Communicated by Richard Palais, November 14, 1969

In this paper we extend almost all the results on infinite-dimensional Fréchet manifolds to apply to manifolds modeled on some $l_{2}^{f}\left(=\left\{x \in l_{2} \mid\right.\right.$ at most finitely many of the coordinates of $x$ are nonzero $\}$ ) and we show (Theorem 14) that each $l_{2}^{\dagger}$-manifold has a unique completion to an $l_{2}$-manifold. (We use $l_{2}$ to stand for the Hilbert space of all square-summable sequences of some infinite cardinality $\mathfrak{A}$. When we wish to be more specific, we write $l_{2}(\mathfrak{A})$.) Examples of $l_{2}^{f}\left(\boldsymbol{\aleph}_{0}\right)$-manifolds include the metric $S^{\infty}$ (the unit sphere in $l_{2}^{f}\left(\boldsymbol{\aleph}_{0}\right)$ ) and the metric $R P^{\infty}$, which may be regarded as the orbit space of $S^{\infty}$ acted upon by the antipodal map. (The identity map from $S^{\infty}$ or $R P^{\infty}$ with the weak topology to the metric topology is a homotopy equivalence. (see [2]).) See also Theorem 16. In fact, we show (Theorems 15 and 17) that each $l_{2}^{\dagger}$-manifold is a metric (see [2] for definition) simplicial complex and that each $l_{2}^{f}\left(\boldsymbol{\aleph}_{0}\right)$-manifold is the 'metric' direct limit of finite-dimensional, closed, orientable manifolds. We conjecture that the metric geometric realization of each connected singular s.s. complex (or Kan s.s. complex) is an $l_{2}^{f}(\mathfrak{H})$-manifold, for some cardinal $\mathfrak{A}$. Most of the results here have been independently proved for the case of $l_{2}^{f}\left(\boldsymbol{\aleph}_{0}\right)$ by T. A. Chapman [1] who used different methods. All manifolds in this paper are assumed paracompact.

Definitions. (1) If $F$ is a TVS, define $F^{\omega}$ to be the countably infinite product and $F_{f}^{\omega}=\left\{\left\{x_{i}\right\} \in F^{\omega} \mid\right.$ for at most finitely many $\left.i, x_{i} \neq 0\right\}$.

(2) Let $X$ and $Y$ be spaces, $\mathcal{U}$ be an open cover of $Y$, and $\mathcal{F}$ a set of functions from $X$ into $Y$. Then $\mathcal{F}$ is said to be $U$-small if for each $x \in X$ there is a $U \in \mathcal{U}$ containing $\{f(x) \mid f \in F\}$. Members of $F$ are said to be $\mathcal{U}$-approximate. A homotopy $F: X \times I \rightarrow Y$ is said to be $\mathcal{U}$-small if $\mathcal{F}=\{F(, t) \mid t \in I\}$ is. A function $g: X \rightarrow Y$ is said to be approximated

AMS Subject Classifications. Primary 5755; Secondary 5525.

Key Words and Phrases. Metric simplicial complex, triangulated infinite-dimensional manifold, contractible homeomorphism space, trivial micro-bundle, stable infinite-dimensional manifold, open embedding, embedding-approximated map, topological classification, homotopy type, negligible $Z$-set, metric direct limit.

1 Alfred P. Sloan fellow.

${ }^{2}$ Partially supported by NSF Grant GP 9397. 
by the set $\mathfrak{F}$ if for each $\mathcal{U}$ there is a member $f$ of $\mathfrak{F}$ such that $f$ and $g$ are $\mathcal{U}$-approximate.

(3) Two embeddings $f, g: X \rightarrow Y$ are ambient invertibly isotopic if there is a level-preserving homeomorphism (called an invertible isotopy) $h: Y \times I \rightarrow Y \times I$ such that $h(y, 0)=(y, 0)$ for each $y \in Y$, and $h(f(x), 1)=(g(x), 1)$ for each $x \in X$.

(4) A pair $(M, N)$ is an $\left(l_{2} l_{2}^{f},\right)$-manifold pair if $M$ is an $l_{2}$-manifold for which there is an open cover $u$ and open embeddings $\left\{f_{U}: U \rightarrow l_{2} \mid U \in \mathcal{u}\right\}$ such that for each $U \in \mathcal{u}, f_{U}(U \cap N)=f_{U}(U) \cap l_{2}$.

THEOREM 1. If $(M, N)$ is an $\left(l_{2}, l_{2}^{f}\right)$-manifold pair, then the inclusion of $N$ into $M$ is a homotopy equivalence.

Proof. Lemma 4 and Theorem 6 of [13] (which contains a topological characterization of $\left(l_{2}, l_{2}^{f}\right)$-manifold pairs) ensure that it induces isomorphisms on all homotopy groups, since any map of a sphere into $M$ or $N$ is homotopic to an embedding; and because $M$ and $N$ are ANR's for metric spaces it must be a homotopy equivalence (see [9]).

THEOREM 2 (UNIQUENESS OF $\left(l_{2}, l_{2}^{f}\right)$-MANIFOLD PAIRS). Let $M$ be an $l_{2}$-manifold, and let $(M, N)$ and $\left(M, N^{\prime}\right)$ be two $\left(l_{2}, l_{2}^{r}\right)$-manifold pairs. The identity map of $(M, N)$ is approximated by homeomorphisms of the pair $(M, N)$ onto $\left(M, N^{\prime}\right)$ which are ambient isotopic to it by $u$-small isotopies for all $u$.

Proof. This is part of Theorem 6 of [13].

Corollary 1. $\left(l_{2}, l_{2}^{f}\right)$ is homeomorphic as a pair to $\left(\left(l_{2}\right)^{\omega},\left(l_{2}^{f}\right)_{f}^{\omega}\right)$.

Proof. This is the relative statement of Corollary 3 of [13]. The proof given there, however, coupled with Theorem 2 gives the result.

Note that $l_{2}^{f}$ (or $\left.\left(l_{2}^{f}\right)_{f}^{\omega}\right)$ satisfies the hypotheses of Theorems 3-12.

Theorem 3. (Homeomorphism SPACES ARE CONTRACTIBLE). Let $F$ be a TVS which is homeomorphic to $F^{\omega}$ or $F_{f}^{\omega}$, then the space (C-O topology) of homeomorphisms of $F$ is contractible.

Proof. If $F \cong F^{\omega}$ this is proved by Renz in [10], but an inspection of Renz's proof shows that it works with $F^{\omega}$ replaced by $F_{f}^{\omega}$. (Note that, if $F \cong F_{f}^{\omega}$, then $F \cong F \times F$.)

TheOREM 4 (MICRo-BUNdLES ARE TRIVIAL). Let $F$ be a TVS which is homeomorphic to $F^{\omega}$ or $F_{f}^{\omega}$ and let $B$ be a paracompact space with the homotopy-type of a simplicial (or CW) complex. Then every microbundle with base $B$ and fiber $F$ is trivial. 
ProoF. If $F \cong F^{\omega}$ this is proved by Henderson in [6]. The only place in [6] in which the hypothesis $F \cong F^{\omega}$ is used is in Lemma 4.2 which is proved in Renz's paper [10]. As above an inspection of Renz's proof shows that the hypothesis $F \cong F^{\omega}$ may be replaced by $F \cong F_{f}^{\omega}$.

ThEOREM 5 (MANIFOLDS ARE STABLE). Let $F$ be a metrizable TVS which is homeomorphic to $F^{\omega}$ or $F_{f}^{\omega}$. If $M$ is a manifold modeled on $F$, then $M \times F \cong M$.

Proof. This theorem was proved by R. Schori in [11, Theorem 5.4] when $F \cong F^{\omega}$. As above, for $F \cong F_{f}^{\omega}$ apply the arguments of the proof of Theorem 5.4 of [11] to the TVS, $F$, and note that all maps involving $F^{\omega}$ can be restricted to $F_{f}^{\omega}$. Otherwise, Schori's proof goes through without change.

Theorem 6 (Closed embedding THeOREM). Let $F$ be a metrizable $T V S$ which is homeomorphic to $F^{\omega}$ or $F_{f}^{\omega}$. If $M$ is a connected manifold modeled on $F$, then $M$ can be embedded as a closed submanifold of $F$.

Proof. The proof is the same as the proof of Theorem 1 of [6], except that when $F \cong F_{f}^{\omega}$ one must note that, since $\left\{V_{\alpha}\right\}$ is locally finite, $\tilde{h}_{i}(m)$ is zero for all but finitely many $i$ 's. Therefore $h$ is an embedding

$$
h: M \rightarrow(F \times F)_{f}^{\omega} \cong F .
$$

THEOREM 7 (OPEN EMBEDDING THEOREM). Let $F$ be a metrizable locally-convex TVS (MLCTVS) which is homeomorphic to $F^{\omega}$ or $F_{f}^{\omega}$. Each connected manifold, $M$, modeled on $F$ can be embedded as an open subset of $F$.

Proof. Follows immediately from Theorem 5 of this paper and Theorem 2 of [7], because Theorem 4 of this paper allows the hypothesis, " $F \cong F^{\omega}$," of Theorem 2 of [7] to be changed to " $F \cong F_{f}^{w}$ ".

CoRollary 2. For $M$ and $F$ as in Theorem 7, there is an open cover $\mathcal{u}$ of $M$ such that any two $\mathcal{u}$-approximate maps are homotopic by a u-small homotopy.

THEOREM 8. Let $F$ and $M$ be as in Theorem 7. Then the projection $p_{1}: M \times F \rightarrow M$ can be approximated by homeomorphisms $h: M \times F \rightarrow M$.

Proof. This follows from Schori's proof of Corollary 2.3 of [11] with the same modification as in the proof of Theorem 5 .

Theorem 9 (Approximating maps By embeddings). Let $M$ and $N$ be manifolds modeled on a MLCTVS F which is homeomorphic to $F^{w}$ 
or $F_{f}^{\omega}$. Then each map $f: M \rightarrow N$ can be approximated by closed embeddings $h_{1}: M \rightarrow N$ and open embeddings $h_{2}: M \rightarrow N$.

Proof. Same as the proof of Theorem B of [8] because as noted above Theorem $\mathrm{H} 3$ of [8] which is Theorem 2 of [6] applies in this case.

Theorem 10 (Ambient isotopy theorem). Let $N$ be a manifold modeled on the normed TVS, $F$, which is homeomorphic to $F^{\omega}$ or $F_{f}^{\omega}$. If $f, g: X \rightarrow N \times\{0\} \subset N \times F$ are homotopic closed embeddings of an $A N R$ (for metric spaces), then $f$ and $g$ are ambient invertibly isotopic in $N \times F$.

The proof is the same as Theorem 3 of [7] with obvious modifications as above.

Theorem 11 (Classification By homotopy tyPe). Let $F$ be as in Theorem 10 and let $M$ and $N$ be manifolds modeled on $F$. Then each homotopy equivalence between $M$ and $N$ is homotopic to a homeomorphism.

The proofs of Theorems 11 and 12 are the same as Theorems $\mathrm{C}$ and $\mathrm{D}$ of [8] with modifications as above. Following R. D. Anderson we say that a subset $K$ of a space $X$ has Property $Z$ in $X$ if, for each nonempty, homotopically-trivial open set $U \subset X, U-K$ is nonempty and homotopically trivial.

TheOREM 12 ( $Z$-SETS ARE NEGLigible). Let $F$ and $N$ be as in Theorem 10. If $K$ is a closed set with Property $Z$ in $N$, then $K$ is negligible, that is, $N-K$ is homeomorphic to $N$. In fact, the homeomorphism is homotopic to the inclusion $N-K \rightarrow N$.

THEOREM 13. If $K$ is a locally finite-dimensional simplicial complex with the barycentric metric such that no star contains more than $\mathfrak{A}$ vertices, then $\left(K \times l_{2}(\mathfrak{A}), K \times l_{2}^{f}(\mathfrak{A})\right)$ is an $\left(l_{2}(\mathfrak{H}), l_{2}^{f}(\mathfrak{U})\right)$-manifold pair.

Proof. See [13, Corollary 6].

Theorem 14 (Completions of $l_{2}^{t}$-MANifolds). If $N$ is a manifold modeled on $l_{2}^{f}$, then there is an $\left(l_{2}, l_{2}^{f}\right)$-manifold pair $(M, N)$; it is unique up to homeomorphisms of pairs.

Proof. $N$ must have the homotopy type of a simplicial complex $K$ satisfying the hypotheses of the above theorem (see [12, Theorem 5], or [6, Lemma 3.1]), so $\left(K \times l_{2}, K \times l_{2}^{f}\right)$ is an $\left(l_{2}, l_{2}^{f}\right)$-manifold pair in which $K \times l_{2}^{f}$ is homeomorphic to $N$ by Theorem 11 . The uniqueness is Theorem 1 followed by Theorem 11 (applied to the completion) followed by Theorem 2 . 
ThEOREM 15 (TRIANGULATION of $l_{2}^{f}$-MANIFOLDS). Each $l_{2}^{\dagger}$-manifold is homeomorphic to a metric simplicial complex.

PRoof. As in the proof of Theorem 14, each $l_{2}^{t}$-manifold is homeomorphic to $K \times l_{2}^{f}$, for some metric simplicial complex $K$, but it is not difficult to show that $l_{2}^{f}$ has a triangulation as a metric simplicial complex and (see [2]) that products of metric simplicial complexes are metric simplicial complexes.

Definition. A metric direct limit space (system) is a direct limit space (system) in the category of metric spaces and isometries. Each metric space is the metric direct limit of any cover of itself which is closed under finite unions (with the maps being the inclusions).

THEOREM 16. If $M^{1} \subset M^{2} \subset \cdots \subset M^{n} \cdots$ is a sequence of metrizable manifolds $\left(\operatorname{dim}\left(M^{n}\right)=n\right)$ without boundary, each bicollared in the next, then the manifolds may be metrized so that it is a metric direct system whose limit is an $l_{2}^{f}\left(\boldsymbol{\aleph}_{0}\right)$-manifold of the same homotopy type as the weak direct limit.

REMARK. It is easy to construct such a sequence which has some metrics with respect to which the metric direct limit does not have the homotopy type of any $l_{2}^{f}\left(\boldsymbol{\aleph}_{0}\right)$-manifold.

Proof. An examination shows that the proof of 5.3 on pp. 186-187 of [3] establishes that any equivalent metric on a closed subset of a metric space may be extended to an equivalent metric on the entire space. An inductive application of this metrizes $M^{n+1}$ so that the inclusion $i_{n}$ of $M^{n}$, regarded as $M^{n} \times\{0\}$, into $M^{n+1}$ extends to an isometric embedding $i_{n}$ of $M^{n} \times(-1,1)$. Then $M^{1} \subset M^{2} \subset \cdots \subset M^{n}$ ... is a metric direct system whose limit $M$ is an $l_{2}^{f}\left(\boldsymbol{\aleph}_{0}\right)$-manifold. (This may be seen since if $x \in M^{n}$ and $U$ is an open neighborhood of $x$ homeomorphic to $R^{n}$, then $U \times\left\{\left(x_{1}, \cdots\right) \in l_{2}^{\prime}|| x_{i} \mid<1\right.$, for all $\left.i\right\}$ is isometric with inj $\lim \left\{i_{n+m}\left(U \times(-1,1)_{1} \times \cdots \times(-1,1)_{m}\right\}\right.$, a neighborhood of inj $\lim \{x\}$ in $M$.)

To see that $M$ is of the same homotopy type as $M_{w}$, the weak direct limit, it is only necessary to show that $M$ and $M_{w}$ have the same weak homotopy type, since they are ANR's. (See [9].) Regarding $M$ and $M_{w}$ as two different topologizations of the same underlying set and observing that the image of any map $f$ of a compact space into $M$ lies in one of the $M^{n}$ 's together with its successive collars (so that $f$ is homotopic, relative to $M^{n}$, to a map into $M^{n}$ ), it is easy to see that the identity map of $M_{w}$ to $M$ is a weak homotopy equivalence.

THEOREM 17. Each separable $l_{2}^{f}\left(\boldsymbol{\aleph}_{0}\right)$-manifold $M$ is the metric direct limit of a sequence $M^{1} \subset M^{2} \subset \cdots \subset M^{n} \cdots$ of closed, orientable manifolds $\left(\operatorname{dim}\left(M^{n}\right)=n\right)$ each bicollared in its successor. 
Proof. $M$ has the homotopy type of a countable, locally finite, simplicial complex $K$. (For example, see [5].) Let $K$ be embedded in $l_{2}^{\prime}\left(\boldsymbol{\aleph}_{0}\right)$ simplicially by sending vertices to points of the standard orthonormal basis, and let $L$ be a triangulation (with the norm-induced topology) of $l_{2}^{f}\left(\boldsymbol{\aleph}_{0}\right)$ such that $K$ and each $R^{n}$ (the span of the first $n$ basis elements) is a subcomplex. Define $N$ and $\partial N$ to be the subcomplexes of $L^{\prime \prime}$ (the second barycentric subdivision of $L$ ) composed respectively of all simplices which are faces of simplices meeting $K$ and all such simplices which do not themselves meet $K$.

By standard regular neighborhood theory, $N \cap R^{n}$ and $\partial N \cap R^{n}$ are manifolds bicollared in $N \cap R^{n+1}$ and in $\partial N \cap R^{n+1}, \partial N \cap R^{n}$ is closed and orientable, $K$ is a deformation retract of $N$, and $\partial N$ is a deformation retract of $N-K$. Also, it may be shown directly or through [4] that $N-K$ is of the same homotopy type as $N$, so $\partial N$ has the homotopy type of M. Dowker's theorem [2] then asserts that this is true of $\partial N$ under the weak topology. By Theorem $16, \partial N$ may be remetrized to give an $l_{2}^{f}\left(\boldsymbol{\aleph}_{0}\right)$-manifold, homotopy equivalent to $M$, which is the metric direct limit of $\left\{\partial N \cap R^{n}\right\}_{n=1}^{\infty}$, and by Theorem 11 it is homeomorphic to $M$.

\section{REFERENCES}

1. T. A. Chapman, Four classes of separable metric infinite-dimensional manifolds, Bull. Amer. Math. Soc. (to appear).

2. C. H. Dowker, Topology of metric complexes, Amer. J. Math. 74 (1952), 555-577. MR 13, 965.

3. J. Dugundji, Topology, Allyn \& Bacon, Boston, Mass., 1966. MR 33 \#1824.

4. J. Eells and N. H. Kuiper, Homotopy negligible subsets, Compositio Math. 21 (1969), 155-161.

5. D. W. Henderson, Infinite-dimensional manifolds, Proc. Internat. Sympos. Topology and its Applications, Herceg Novi, Yugoslavia, 1968.

6. - Micro-bundles with infinite-dimensional fibers are trivial (to appear).

7. - Stable classification of infinite-dimensional manifolds by homotopy type, to (appear).

8. D. W. Henderson and R. Schori, Topological classification of infinite-dimensional manifolds by homotopy type, Bull. Amer. Math. Soc. 76 (1970), 121-124.

9. R. S. Palais, Homotopy theory of infinitedimensional manifolds, Topology $\mathbf{5}$ (1966), 1-16. MR 32 \#6455.

10. P. L. Renz, The contractibility of the homeomorphism group of some product spaces by Wong's method (to appear).

11. R. Schori, Topological stability for infinite-dimensional manifolds (to appear).

12. J. E. West, Products of complexes and Fréchet spaces which are manifolds (to appear).

13. - The ambient homeomorphy of an incomplete subspace of Hilbert spaces, Pac. J. Math. (to appear).

Cornell University, IthacA, New York 14850 